proceedings

\title{
Spatial Analysis of the Flight Paths of Pigeons over Urban and Natural Terrain ${ }^{+}$
}

\author{
Margarita Zaleshina ${ }^{1}$ and Alexander Zaleshin ${ }^{2, *}$ \\ 1 Moscow Institute of Physics and Technology, Moscow, Russia; zaleshina@gmail.com \\ 2 Institute of Higher Nervous Activity and Neurophysiology, Moscow, Russia \\ * Correspondence: terbiosorg@gmail.com \\ + Presented at the 1st International Electronic Conference on Brain Sciences, 10-25 November 2020; \\ Available online: https://sciforum.net/conference/IECBS.
}

Received: date; Accepted: date; Published: date

\begin{abstract}
Visual characteristics of terrain affect the properties of pigeon trajectories in mediumdistance flights. Pigeon flight often provides a solution to the task of searching for food (foraging), returning home (homing), or exploring territory (surveying). In this work, we considered the flights of single pigeons and pigeon flocks, calculated flight characteristics such as direction, altitude and its deviations, and analyzed reactions to the boundaries between different areas. Based on remote sensing datasets, we identified visual characteristics of terrain, such as the density of surface fill and its distribution over the study terrain, boundaries of single objects, and boundaries between homogeneous areas. Applying spatial analysis, we compared the characteristics of pigeon GPS tracks and features of object distributions on terrain over which birds fly. Our analysis revealed which flight parameters are stable and which, on the contrary, are very sensitive to visually perceived terrain characteristics. We found that the properties of flight over an urbanized area often differ from the properties of flight over a natural landscape. Spatial data-pigeon GPS track records and open-access remote sensing datasets - were processed. Results show that adaptive visual perception can help solve navigation tasks when pigeons fly over mixed terrain.
\end{abstract}

Keywords: visual perception; spatial navigation; wayfinding

\section{Introduction}

When a pigeon flies over various landscapes, visual landmarks can be perceived in whole as point objects, or as extended linear or areal objects. The characteristic features perceived in landmarks are their borders and textures. Moreover, the textures themselves can consist of elements (patterns), which can be perceived as separate objects at a certain scale of perception. The level of reaction to a visual object can change. A visually noticeable object, at a certain level of attention, can become insignificant, "invisible": that is, a bird stops responding to it. Pigeons are able to quickly respond to changes in the properties of the terrain over which they fly. For orientation, birds can use visual landmarks, such as noticeable single objects or extended lines (roads, rivers, coastlines), or homogeneous surfaces (forests, fields, lakes).

Depending on the type of terrain - urban, natural, or mixed - the visual attributes of the surface change noticeably, including the density and distance between main types of surface elements. In contrast to a reaction to a landmark, which is always perceived as a visually significant object, a reaction to similar elements can differ: elements may be perceived as a unique object or as part of a texture pattern. For a natural area, the characteristic size of a texture element is about a meter to a dozen meters. For an urbanized area, the typical size of an individual object is measured in hundreds of meters. Therefore, it can be assumed that with visual perception during flight over a forest, most of observed elements for a pigeon will form a homogeneous texture. When flying over an urbanized Proceedings 2020, 4, $\mathrm{x}$; doi: FOR PEER REVIEW www.mdpi.com/journal/proceedings 
terrain, each house from a residential area will be perceived as a separate element. In another case, a detached house in a forest can become an object of interest or merge with the texture, depending on the scale of perception at this point. This perception can be reflected in a change of pigeon flight path.

Functionally, the flight of a pigeon most often provides a solution to the task of searching for food (foraging) or the task of returning home (homing). In addition, pigeons regularly make exploratory flights (surveying). During flight a pigeon can change its speed, direction, and altitude based on varying visual information. In a flock of pigeons, the methods of grouping birds can also change: pigeons in small flocks coordinate their behavior with that of their nearest neighbors, which can change during the flight [1]. Although the shortest distance through the air between two points is a straight line, excluding the effect of wind, birds never fly strictly "in a straight line". A real pigeon flight contains several components, the bird needs to follow the "straight line" direction, or to respond to landmarks. Blaser et al. [2] proved that pigeons are able to remember routes and fly to the objects which are important to them, such as home or feeding spots. The authors suggested that pigeons have their own spatial representation of their surroundings, a so-called "mental map", in which their own position, home loft and food spots are represented simultaneously. In pigeon trajectories, the important components are: (i) beeline path (straight line between start and finish points), (ii) spontaneous deviations or deviations due to wind (Figure 1).

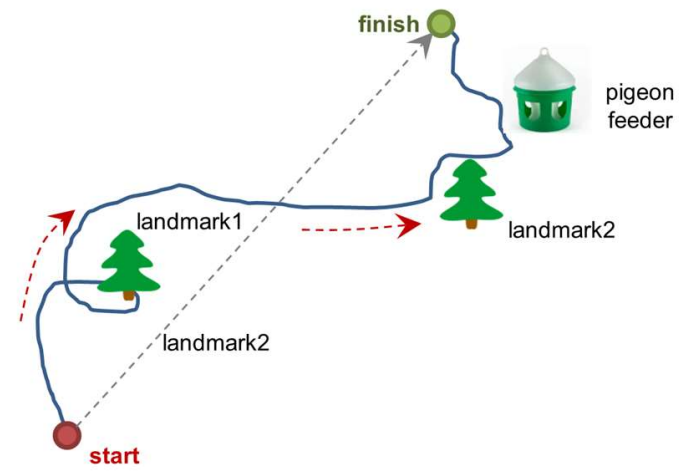

Figure 1. Pigeon flight path in relation to external landmarks. Blue line shows pigeon's track, dashed gray line shows a beeline to final point in a straight direction, dashed red lines show typical direction in single time intervals.

A study of perception of the surrounding landscape during the flight and the influence of landscape on wayfinding and training of pigeons during the flight demonstrated that memorized guidance control is likely to relate to local features of the landscape [3]. Lipp et al. [4] discovered that pigeons can select extended linear objects and use them as landmarks. Biro et al. [5] reported similar results: pigeons can fly home in a straight line, but more often they fly along well-known routes, preferring to be guided by familiar landmarks. Moreover, near their loft, pigeons mainly orient on familiar visual landmarks [6]. Schiffner et al. [7] consider that differences in correlation dimension, a variable reflecting the complexity of the navigational process, reflect differences in the use of navigational information, with one bird apparently using less complex information than others.

\section{Materials and Methods}

This paper shows the characteristic features of pigeon flights over medium distances (about 10 $\mathrm{km}$ ) over various landscapes. We calculated and analyzed the following datasets: (i) spatial features of types of terrain over which pigeon flights occur, (ii) features of pigeon flights and their variation and sustainability, and (iii) comparison of spatial features of terrain and features of pigeon flights.

Flight trajectories of poorly trained individual pigeons and pigeons in flocks were studied. Pigeons flew (i) over natural and agricultural areas (the typical distance between separate surface elements was assumed to be more than $100 \mathrm{~m}$ for fill patterns and less than $10 \mathrm{~m}$ for single elements), (ii) over urban areas with low-rise buildings (the typical distance between separate surface elements was assumed to 
be from $10 \mathrm{~m}$ to $200 \mathrm{~m}$ ), and (iii) over boundaries between different areas. Pigeon flights were performed between two different points with a characteristic distance between them of about 10-15 km, and with departure from the selected starting points and arrival at the pigeon-lofts.

Spatial analysis of individual pigeon trajectories was performed based on Dryad data package (https://doi.org/10.5061/dryad.53f4b, [14]). Calculation was made for 102 flights of individual pigeons. Spatial analysis of flock trajectories was performed based on Dryad data package (https://datadryad.org/resource/doi:10.5061/dryad.f9n8t, [15]) and on Movebank data package (https://www.datarepository.movebank.org/handle/10255/move.365, [16]). Coordinates of separate points on GPS tracks were measured 1-4 times per second.

Flight parameters were recorded using GPS-receivers mounted on pigeons and calculated using QGIS tools (http://qgis.org). Earth remote sensing data-satellite images-in the form of OpenLayers (http://openlayers.org) were applied as a source of information about terrain surfaces. GPS data of pigeon flight paths were processed with precise reference to locations and terrain features. The remote sensing data layers were added using the OpenLayers Plugin. Based on data from the remote sensing datasets, we identified visual characteristics of the terrain, such as the density of surface fill and its distribution over the study area, the boundaries of distinct objects, and the boundaries between homogeneous areas.

The following operations were performed:

Step 1. Segregation of homogeneous textures and borders between textures. The selection of a distinct homogeneous area was performed using the calculation of average density of texture elements of an area. Between selected areas with different textures, boundaries were constructed in the form of isolines. Suitable average density parameters were identified for constructing isoline contours, that make it possible to distinguish forests and fields in the form of extended homogeneous surfaces interspersed with separate inhomogeneous sections. The accuracy of the isolines was set within five meters or more (Figure 4). With the same applied average density parameters, houses in the city were distinguished as independent elements.

Step 2. Calculating the surface fill density distribution and separation of territories by type of surfaces. Calculation of the distribution of surface fill densities was performed on a layer of objects selected by isolines in Step 1. Each selected object was presented as a centroid. According to the density of available centroids (the number of centroids per unit area), the territories over which the pigeons flew were divided into areas with different types of surfaces. The following factors were taken into account: the fill density of textures with similar repeating patterns, the characteristic sizes of simple objects included in these patterns and the typical distances between these simple objects.

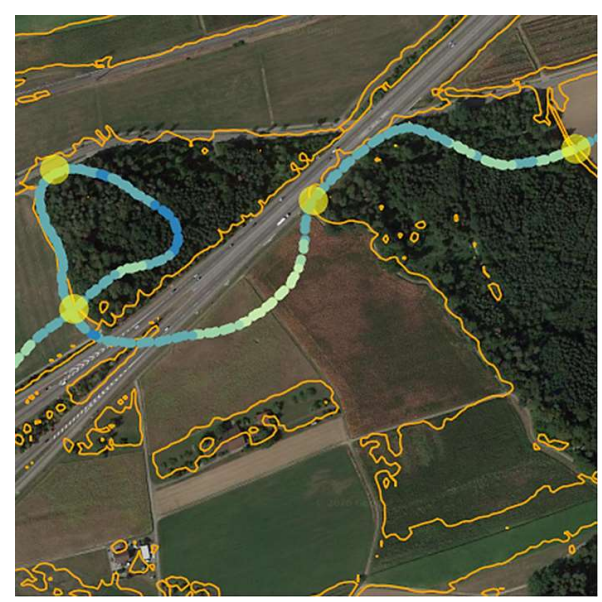

Figure 2. Characteristic features. Blue graded color shows the trajectory of pigeons. Orange lines show the calculated isolines. Yellow circles show intersections of isolines and GPS tracks.

As a result, the surfaces were divided as follows: urban area with low-rise buildings: extended boundaries (rugged or smooth) of linear objects or boundaries between textures, with an average length of more than $300 \mathrm{~m}$; natural area (forest, grassland, fields); other territories. 
Based on data records of GPS tracks, we identified characteristics of pigeon flights related to changes in bird flight parameters (direction and height relative to the altitude of the arrival point). The following operations were performed for all flights of pigeons:

- Calculation of pigeon flight direction based on coordinates for each moment of the GPS track and calculation of direction variability within one second.

- Calculation of height variability within one second.

- Calculation of stability or variability of heights and directions within five seconds.

When assessing the maximum and minimum variability in direction, cases with values of more than 60 degrees and less than 2 degrees were removed from the analysis as artifacts.

\section{Results}

In this study, 137 flights of pigeons were analyzed, including 25 flights in flocks of pigeons. The number of points in GPS records for individual pigeons ranged from 1600 to 7400 . Pigeons flew over a mixed territory composed of forests, agricultural fields and urban territory with low-rise buildings. The most widely presented ranges of parameter values for pigeon flights were identified by characteristics such as direction and altitude and their deviations. For each pigeon flight, the following characteristics were analyzed: instant flight direction (at one point of GPS recording), change in flight direction in an interval of five seconds, instant flight altitude (at one point of GPS recording), and change in flight altitude in an interval of five seconds. It was assumed that:

- Flight can be considered stable in direction if deviations from the direction within five seconds do not exceed 8 degrees.

- Flight can be considered highly variable in direction if deviations from the direction within five seconds exceed 20 degrees.

- Flight can be considered low height-adjustable (with a stable perception scale) if the deviation in height for five seconds does not exceed $1 \mathrm{~m}$.

- Flight can be considered high height- adjustable (with a change in the scale of perception) if the deviation in height for five seconds exceeds $2 \mathrm{~m}$.

In particular, it was shown that there are characteristics in which flight can be considered stable in parameters for an interval of five seconds, and characteristics in which flight parameters change sharply. Then for each type of territory percentages were determined as the ratio of flight time with considered features to the total flight time with an interval of ten seconds. Thus, the combined indicators typical for flights over a certain type of territory were revealed.

Table 1 shows flight features calculated on the base of GPS tracks, such as stable direction (d_stab1,2), direction variability (d_var1,2), low height-adjustability (h_stab1,2) and high heightadjustability (h_var1,2), where preliminary data sources were GPS records from Movebank (MVB) and Dryad (DRD) packages.

Table 1. Comparison of characteristics of pigeon flights and terrain features.

\begin{tabular}{cccccc}
\hline & Indicator & Urban & Field & Boundary & Forest \\
\hline MVB & d_stab1 & 0,10 & 0,20 & 0,31 & 0,29 \\
& d_var1 & 0,12 & 0,19 & 0,36 & 0,17 \\
& h_stab1 & 0,26 & 0,23 & 0,21 & 0,34 \\
& h_var1 & 0,14 & 0,24 & 0,25 & 0,28 \\
\hline DRD & d_stab2 & 0,07 & 0,18 & 0,38 & 0,26 \\
& d_var2 & 0,10 & 0,16 & 0,34 & 0,12 \\
& h_stab2 & 0,24 & 0,21 & 0,22 & 0,31 \\
& h_var2 & 0,17 & 0,25 & 0,27 & 0,23 \\
\hline
\end{tabular}

Using the methods of multi-factor analysis, it was found that the percent ratio was significantly differs for different types of territories and for flight features considered in Table 1 . The analysis of 
variance (ANOVA) was used to detect significant factors in a multi-factor model. According to selected indicators, the dependence of the flight characteristics on the terrain over which the flight takes place had a statistically significant effect $(\mathrm{F}=6.81$, FCritical $=3.07, p<0.05)$. At the same time, it was found that for single characteristics, it was impossible to reliably compare the flight parameters and the area over which the pigeon flies. All factors were checked in pairs against each other on the full flight recording time for the absence of dependence. For additional verification, according to GPS tracks for a control group of 15 pigeons, flight indicators were calculated with a sliding window of ten seconds. For each control point of the GPS track, a preliminary assumption was made about type of terrain over which the pigeons flew. The results of determining the type of terrain by preliminary calculation were compared with data on the type of real terrain.

Further, each indicator from the list $\left\{d_{-}\right.$stab, d_var, h_stab, h_var\} was assigned a value of 0 if it is less than 0.21 , or a value of 1 if it is more than 0.21 . Depending on the total composition of the territory, i.e., on the ratio of different types of homogeneous and heterogeneous textures present in it, the algorithm for assigning 0 and 1 must be re-calculated. For future calculation we set for input arrays [d_stab d_var h_stab h_var] following output values: $[0,0,1,0]=$ Urban; $[0,0,1,1]=$ Field; $[1,1,1,1]$ $=$ Boundary; $[1,0,1,1]=$ Forest. It was found that the accuracy of prediction of the type of terrain, over which the pigeons flew, according to the combined parameters of the flight of pigeons, is $38 \%$. An example of forest identification is shown in Figure 3. Green circles mark places that are identified as a forest based on the properties of the pigeon's flight.

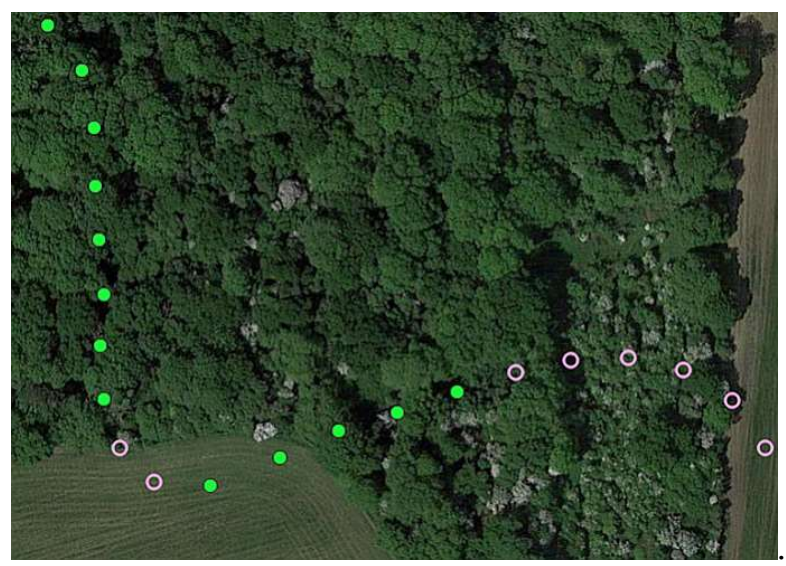

Figure 3. Comparison of the predicted type of terrain and the actual type. Green dots show places that were identified as "forest" based on the properties of the pigeon's flight. Transparent dots show places that were identified as another type of terrain.

\section{Discussion}

The significance of external landmarks and their influence on the change of direction does not only depend on the level of pigeon training. Vyssotski et al. [8] showed that pigeons move straight to the goal from the sea to the shore in good visibility conditions, but in bad visibility conditions they choose the direction of the closest land-mark on the shore. Mann et al. [9] concluded that pigeons orient themselves better when flying above territory where landscape complexity is neither too high nor too low. Visual perception of terrain is important when pigeons fly over medium distances [10]. At the same time, depending on the situation, the level of detail in perception can be changed (by changing the height of the flight, or the distance from objects of interest). That is, the scope of perception is co-scaled to the problem being solved. Visually recognizable extended boundaries between homogeneous textures are important spatial landmarks. Chan et al. [11] argued that extended surfaces or boundaries can act as landmarks by providing a frame of reference for encoding spatial information. In addition, the ability to perceive, distinguish, and respond rapidly to minor changes in textures can be very high in pigeons [12]. The reaction to extended boundaries between different homogeneous textures can be revealed by the flight of pigeons. The elements of a texture 
are defined as visually recognizable items that repeat along the surface. During motion, these elements provide visual information for perception [13].

Depending on the detail level, the same objects can either be perceived separately or merge with the surrounding background. A free-standing tree can serve as a land-mark like a "beacon", but a similar tree in a forest or in a long alley will only be an element within a texture. An array of trees in a forest or even blocks of low-rise buildings can be perceived as a single homogeneous texture with a large coverage.

\section{Conclusions}

Here we studied the typical tasks of finding a flight path over poorly familiar area. Visual spatial recognition can help in the basic formation and quick reconfiguration of flight paths. Based on the calculations made in this work, various cases were identified, for which the flight characteristics of pigeons are stable or sensitive to a change in external environment. Such methods can be applied to study the navigational mechanisms in pigeon flight over mixed terrains. Using the method of multifactor analysis, it was found that in order to compare the flight characteristics of pigeons with the type of territory over which they fly, it is necessary to take into account four factors in GPS track sections: (i) a stable direction for five or more seconds, (ii) a high variability in direction or sharp change in direction, (iii) stable height for five or more seconds, (iv) high variability in height.

This study shows the roles of sustainability (return to the previous value after deviation), sensitivity (variations in flight features near lengthy linear objects or near boundaries of distinct areas), and scale perception (represent itself in change of flight height). The results obtained in this study can be used both for a better understanding of the spatial behavior of living creatures (humans and animals) and for optimization of artificial intelligence algorithms.

Author Contributions: Conceptualization, A.Z. and M.Z.; methodology, A.Z.; software, A.Z. and M.Z.; data curation, A.Z.; visualization, M.Z. All authors have read and agreed to the published version of the manuscript.

Funding: This research received no external funding.

Conflicts of Interest: The authors declare no conflict of interest.

\section{References}

1. Chen, D.; Xu, B.; Zhu, T.; Zhang, H.-T.; Zhou, T. Anisotropic interaction rules in circular motions of pigeon flocks: An empirical study based on sparse Bayesian learning. Phys. Rev. E 2017, 96, 022411.

2. Blaser, N.; Dell'Omo, G.; Dell'Ariccia, G.; Wolfer, D.P.; Lipp, H.-P. Testing cognitive navigation in unknown territories: Homing pigeons choose different targets. J. Exp. Biol. 2013, 216, 3123-3131.

3. Guilford, T.; Biro, D. Route following and the pigeon's familiar area map. J. Exp. Biol. 2014, 217, 169-179.

4. Lipp, H.-P.; Vyssotski, A.L.; Wolfer, D.P.; Renaudineau, S.; Savini, M.; Tröster, G.; Dell'Omo, G. Pigeon Homing along Highways and Exits. Curr. Biol. 2004, 14, 1239-1249.

5. Biro, D.; Freeman, R.; Meade, J.; Roberts, S.; Guilford, T. Pigeons combine compass and landmark guidance in familiar route navigation. Proc. Natl. Acad. Sci. USA 2007, 104, 7471-7476.

6. Walcott, C. Multi-modal orientation cues in homing pigeons. Integr. Comp. Biol. 2005, 45, 574-581.

7. Schiffner, I.; Fuhrmann, P.; Reimann, J.; Wiltschko, R. Behavioural traits of individual homing pigeons, Columba livia f. domestica, in their homing flights. PLoS ONE 2018, 13, e0201291.

8. Vyssotski, A.L.; Dell'Omo, G.; Dell'Ariccia, G.; Abramchuk, A.N.; Serkov, A.N.; Latanov, A.V.; Loizzo, A.; Wolfer, D.P.; Lipp, H.-P. EEG Responses to Visual Landmarks in Flying Pigeons. Curr. Biol. 2009, 19, 11591166.

9. Mann, R.P.; Armstrong, C.; Meade, J.; Freeman, R.; Biro, D.; Guilford, T. Landscape complexity influences route-memory formation in navigating pigeons. Biol. Lett. 2014, 10, 20130885.

10. Zaleshina, M.; Zaleshin, A. Spatial features of terrain reflected in pigeon flights. In German Conference on Spatial Cognition; Springer: Cham, Switzerland, 2018; pp. 3-14.

11. Chan, E.; Baumann, O.; Bellgrove, M.A.; Mattingley, J.B. From objects to landmarks: The function of visual location information in spatial navigation. Front. Psychol. 2012, 3, 1-11.

12. Bovet, D.; Vauclair, J. Picture recognition in animals and humans. Behav. Brain Res. 2000, 109, 143-165. 
13. Zaleshina, M.; Zaleshin, A.; Galvani, A. Visual perception of mixed homogeneous textures in flying pigeons. In International Workshop on Machine Learning, Optimization, and Big Data; Springer: Cham, Switzerland, 2018; pp. 299-308.

14. Pettit, B.; Flack, A.; Freeman, R.; Guilford, T.; Biro, D. Not just passengers: Pigeons, Columba livia, can learn homing routes while flying with a more experienced conspecific. Proc. R. Soc. B Biol. Sci. 2012, 280, 20122160.

15. Watts, I.; Pettit, B.; Nagy, M.; De Perera, T.B.; Biro, D. Lack of experience-based stratification in homing pigeon leadership hierarchies. R. Soc. Open Sci. 2016, 3, 150518.

16. Santos, C.D.; Neupert, S.; Lipp, H.-P.; Wikelski, M.; Dechmann, D.K.N. Temporal and contextual consistency of leadership in homing pigeon flocks. de Polavieja GG, editor. PLoS ONE 2014, 9, e102771.

Publisher's Note: MDPI stays neutral with regard to jurisdictional claims in published maps and institutional affiliations.

(C) 2020 by the authors. Licensee MDPI, Basel, Switzerland. This article is an open access article distributed under the terms and conditions of the Creative Commons Attribution (CC BY) license (http://creativecommons.org/licenses/by/4.0/). 